\title{
Trends in Breast Cancer Incidence in Iraq During the Period 2000-2019
}

\section{Muzahem M Y AL-Hashimi*}

\begin{abstract}
Background: Breast cancer is the most common cancer among women around the world. Objective: This study aims to explore the time trends in the incidence of breast cancer in Iraq in twenty years period (2000 to 2019) to assist health officials and decision-makers in planning and organizing programs to controlling breast cancer growth and enhance women's health. Methods: Breast cancer data from 2000 to 2019 were obtained from the Iraqi Cancer Registry's yearly book series (Ministry of Health). The United Nations Population Division provides annual population estimates by 5-year age groups and gender. Joinpoint regression analysis was used to calculate and assess age-specific and age-standardized incidence rates. Results: A total of 72,022 breast cancer cases were identified among women in Iraq between 2000 and 2019. The average age-standardized incidence rate (ASIRs) was 37.883/100,000. Breast cancer ASIR Iraq exhibited a significantly rising trend during the study period, with an average annual percentage change (AAPC) of $+3.192 \%$. Furthermore, a significant increase in breast cancer incidence for the age group 40-49 (AAPC $=+2.162 \%$ ), 50-59 $(\mathrm{AAPC}=+3.210 \%), 60-69(\mathrm{AAPC}=+5.551 \%)$, and age 70 and over $(\mathrm{AAPC}=+7.943 \%)$ were observed. When compared to other countries in the world, Iraq had a moderate rate of breast cancer ASIR. Conclusions: In summary, our finding revealed an increase in the incidence rates of breast cancer among Iraqi women from 2000 to 2019. Further research is needed to explore risk factors including levels of overweight, dietary changes, physical inactivity, obesity, smoking, high marriage age, and low birth rates to prevent and control breast cancer.
\end{abstract}

Keywords: Breast cancer- incidence- poisson regression- time trends

Asian Pac J Cancer Prev, 22 (12), 3889- 3896

\section{Introduction}

Breast cancer is the most common cancer and the fifth leading cause of cancer mortality in females globally. In 2020, it is estimated that 2.3 million females were diagnosed with breast cancer representing $11.7 \%$ of all cancer cases, and 685,000 deaths representing $6.9 \%$ of all cancer deaths in females (Sung et al., 2021). Incidence rates for breast cancer varied widely across regions (Sung et al., 2021). Females in high/very higher HDI countries have higher breast cancer incidence rates than in low/medium HDI countries (55.9 and 29.7/100,000, respectively), with the highest incidence rates in Australia/New Zealand (95.5/100,000), Western Europe (90.7/100,000), Northern America (89.4/100,000), and Northern Europe $(86.4 / 100,000)$ and the lower rates in South-Eastern Asia (41.2/100,000), Central America (39.5/100,000), Eastern Africa (33.0/100,000), Middle Africa (32.7/100,000), and South Central Asia (26.2/100,000) (Sung et al., 2021).

Breast cancer incidence rates increased rapidly in Northern America, Oceania, and Europe throughout the 1980s and 1990s, most likely due to increases in mammographic screening. Then, in the early 2000 s, the incidence dropped or stabilized (Sung et al., 2021; Torre et al., 2017). On the contrary, it had been increasing fast in Central and South America, Africa, and Asia (Sung et al., 2021; Torre et al., 2017).

It has been estimated that around $20 \%$ of breast cancers globally are caused by modifiable risk factors such as overweight and obesity, alcohol use, and physical inactivity, suggesting that improving healthy behaviors might reduce disease burden (Danaei et al., 2005; Torre et al., 2017).

In Iraq, the trend of the incidence rate of new cases of cancer increased from the year $2000(52.00 / 100,000)$ to the year $2019(91.66 / 100,000)$. Breast cancer is the main cause of mortality among Iraqi women, accounting for about one-third of all cancer cases recorded in the country in 2019 (Iraqi Cancer Board, 2019). The highest percentage and incidence rate of the top ten cancers in 2019 was breast cancer $(34.08 \%, 35.95 / 100,000$ respectively), as well as, it has the highest percentage and mortality rate of cancer (22.58\%, 6.22/100,000 respectively) (Iraqi Cancer Board, 2019).

Ministry of Health $(\mathrm{MOH})$, in cooperation with the Ministry of Higher Education and Scientific Research 
(MOHESR) and World Health Organization (WHO), launched a National Program for Early Detection and Down Staging of Breast Cancer in 2000. Referral centers and specialist clinics for the early diagnosis of breast cancer have since been established in all Iraqi governorates' main hospitals (Al-Alwan and Mualla, 2014). Early Detection and Down Staging is a comprehensive program to provide high quality primary and secondary health services and conduct early detection and examinations for all women aged 20 years and over to reducing mortality rates from breast cancer, moving from diagnosing the disease in its late stages (third and fourth) to diagnose it in the early stages (first and second), where recovery prospects are better, and treatment expenses are lower.

Monitor cancer trends over time are one of the most important functions for health care planning and disease control. This study aims to explore the time trends in the incidence of breast cancer in Iraq in twenty years period (2000 to 2019) to assist health officials and decisionmakers to put in place strategies to controlling breast cancer growth and enhance women's health.

\section{Materials and Methods}

The data of breast cancer (International Classification of Diseases for Oncology (ICD-O-3) code) from 2000 to 2019 were taken from the annual book series published by the Iraqi Cancer Registry (ICR)/Ministry of Health. Every year, the ICR in the Ministry of Health publishes extensive statistical data on cancer in Iraq, including the most common ten cancers and their distribution among governorates, ages, and sexes. The ICR is responsible for collecting information relating to every new cancer patient resident from hospitals and laboratories (public and private) in all directorates of health in Iraq by the CanReg4 program (Iraqi Cancer Board, 2019). The registry was founded in 1974 through close cooperation of the ministry of health and the Iraqi Cancer Society. It began operation in 1975 and was located in the Central Public Health Laboratory. Annual estimates of the female population by 5 -year age group obtained from the United Nations, Population Division, Department of Economic and Social Affairs.

Incidence of breast cancer was represented using agespecific incidence rates (ASR) and age-standardized rates
(ASIRs) (age groups 0-19, 20-29, 30-39, 40-49, 50-59, 60-69, 70+). The ASIRs were calculated using the World standard population. The incidence rate was determined by year, by 5 -year periods, as well as for the whole study period.

Variations in breast cancer incidence rates over time were assessed using the joinpoint regression analysis (version 4.9.0.0). Joinpoint regression was applied to determine the time points essential changes in the trends occurred. The year was used as a predictor variable and log-transformed ASR and ASIR as the response variable. The errors are assumed to have constant variance. The Grid Search Method with uncorrelated errors was used to select the most appropriate number of joinpoints for the data. The minimum number of joinpoints was set to zero and the maximum was set to four. For significance testing, the Monte Carlo Permutation number of joinpoints method was employed. The annual percent change (APC) and average annual percent change (AAPC) in cancer incidence with $95 \%$ CI were computed to evaluate the magnitude and direction of the trends from 2000 to 2019. The significance level was set at a $p$-value $\leq 5 \%$ for all analyses.

\section{Results}

Over the study period 2000-2019, 72,022 cases of breast cancer cases were recorded. Age-specific rates (ASRs) and age-standardized incidence rates (ASIRs) of breast cancer among females in Iraq are presented in Table 1. The average ASIR was 37.883. The AISR for breast cancer incidence increased by $76.516 \%$, with the lowest ASIR $(26.282 / 100,000)$ in the earliest period of 2000-2004, and the greatest AISR $(46.392 / 100,000)$ in the latest period of 2015-2019. The ASIRs of the last two 5 -year periods were significantly higher than those of the first two periods. These rates were 1.586 and 1.765 times higher than the first period (2000-2004).

Figure 1 depicts the yearly incidence rate of breast cancer during the study period. Despite fluctuations from year to year, joinpoint regression analysis indicated a significant increase in the incidence rate across the whole study period. The AAPC was $+3.192 \%(95 \% \mathrm{CI}=+1.368 \%$ to $+5.048 \%$ ), meaning that the ASIR increased by +3.192 percent on average per year. Two joinpoints were detected,

Table 1. Age-Specific and Age-Standardized Incidence Rates (1/100,000) of Breast Cancer among Women in Iraq (2000-2019) by Period

\begin{tabular}{lcccccc}
\hline Age group & \multicolumn{3}{c}{ Period } & & Total & Overall \\
& $2000-2004$ & $2005-2009$ & $2010-2014$ & $2015-2019$ & & changes (\%) \\
\hline $0-19$ & 0.074 & 0.078 & 0.002 & 0.016 & 0.039 & -78.378 \\
$20-29$ & 2.7517 & 3.159 & 4.481 & 4.309 & 3.7683 & 56.594 \\
$30-39$ & 23.927 & 24.22 & 28.957 & 30.761 & 27.449 & 28.562 \\
$40-49$ & 71.118 & 79.53 & 92.769 & 95.576 & 87.275 & 34.391 \\
$50-59$ & 87.761 & 102.444 & 134.815 & 156.529 & 126.037 & 78.358 \\
$60-69$ & 68.612 & 92.291 & 143.529 & 158.477 & 122.218 & 130.976 \\
$70+$ & 37.742 & 53.092 & 93.249 & 125.444 & 81.537 & 232.372 \\
Age-standardized incidence rates (ASIRs) & 26.282 & 31.31 & 41.692 & 46.392 & 37.883 & 76.516 \\
\hline
\end{tabular}


Table 2. Evolution of the Annual Age-Specific Rates of Breast Cancer in Iraq in the Period 2000-2019, 95\% Confidence Intervals in Brackets

\begin{tabular}{lll}
\hline Age group & Joinpoint & Trend APC $(\%)$ \\
\hline $0-19$ & & Not significant; No joinpoint detected \\
$20-29$ & 2003 & $2000-2003:-21.869(-37.083 ;-2.976)$ \\
& 2006 & $2003-2006:+20.575(-21.810 ;+85.938)$ \\
& & $2006-2019:+2.852(+0.543 ;+5.213)$ \\
$30-39$ & 2003 & $2000-2003:-8.911(-16.852 ;-0.213)$ \\
& 2010 & $2003-2010:+2.862(-0.262 ;+6.083)$ \\
$40-49$ & 2011 & $2010-2019:+2.153(+0.466 ;+3.869)$ \\
& & $2000-2011:+2.866(+0.870 ;+4.902)$ \\
$50-59$ & 2003 & $2011-2019:+1.201(-1.958 ;+4.463)$ \\
& 2013 & $2000-2003:-3.089(-11.581 ;+6.218)$ \\
$60-69$ & & $2003-2013:+5.077(+3.332 ;+6.851)$ \\
$70+$ & 2013 & $2013-2019:+3.376(+0.220 ;+6.631)$ \\
& 2004 & $2000-2013:+6.913(+5.147 ;+8.710)$ \\
& & $2013-2019:+2.660(-2.711 ;+8.328)$ \\
& & $2000-2004:+3.323(-10.691 ;+19.537)$ \\
\end{tabular}

2003 and 2012; 2000-2003 (APC -2.375\%; $95 \%$ CI $-11.055,+7.152 \%) ; 2003-2012(\mathrm{APC}+5.388 \% ; 95 \%$ $\mathrm{CI}+3.268,+7.551 \%) ; 2012-2019(\mathrm{APC}+2.850 \%$; 95 $\% \mathrm{CI}+0.322,+5.442 \%)$. The trend changes in the annual incidence between 2000 and 2019 raised by $116.301 \%$.

Breast cancer incidence rates increased with age, with the largest increase was observed in females $70+$ age group (232.372), followed by females aged 60-69 (130.976) (Table 1). The time trends of the ASRs were performed by year. Table 2 and Figure 2 shows the evolution of the annual ASR of breast cancer in Iraq in the period 2000-2019. In the age group 0-19 years, APC decreased statistically not significant over the period 2000-2019 (APC -5.0350\%; 95\% CI, -12.369\%

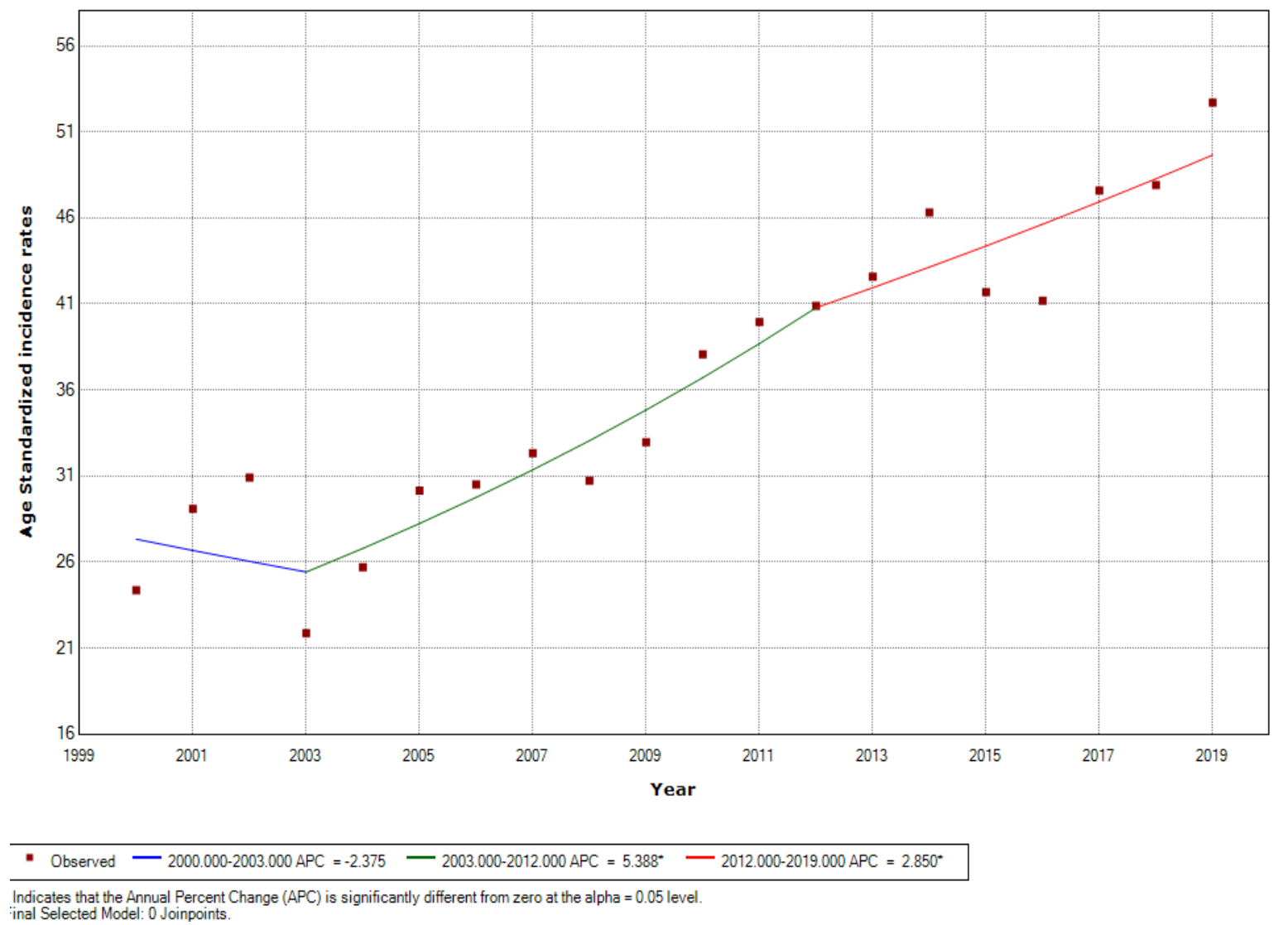

Figure 1. Joinpoint Regression Analysis of Age-Standardized Incidence Rates $(1 / 100,000)$ of Breast Cancer for Iraqi Women, All Ages, 2000-2019. 
All: 2 Joinpoints

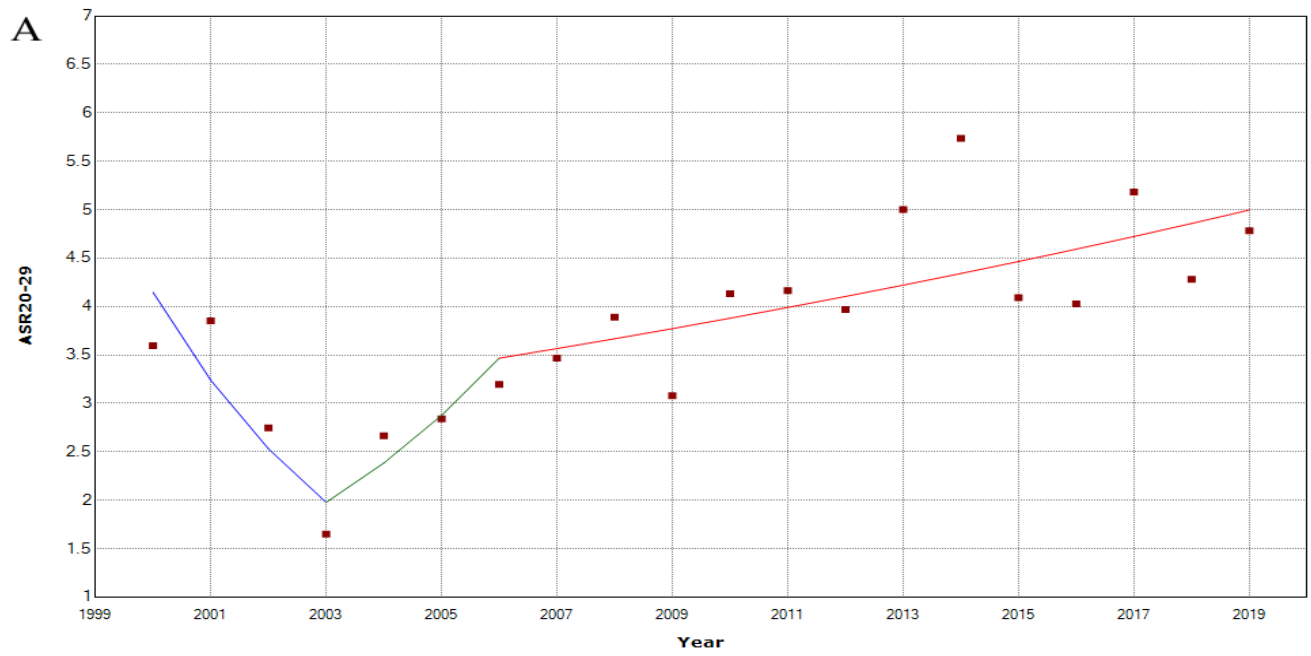

- Observed -2000-2003 APC $=-21.87^{*} \quad$-2003-2006 APC $=20.58 \quad$ - 2006-2019 APC $=2.85^{\circ}$

- Indicates that the Annual Percent Change (APC) is significantly different from zero at the alpha $=0.05$ level.
Final Selected Model: 0 Joinpoints.

All: 2 Joinpoints

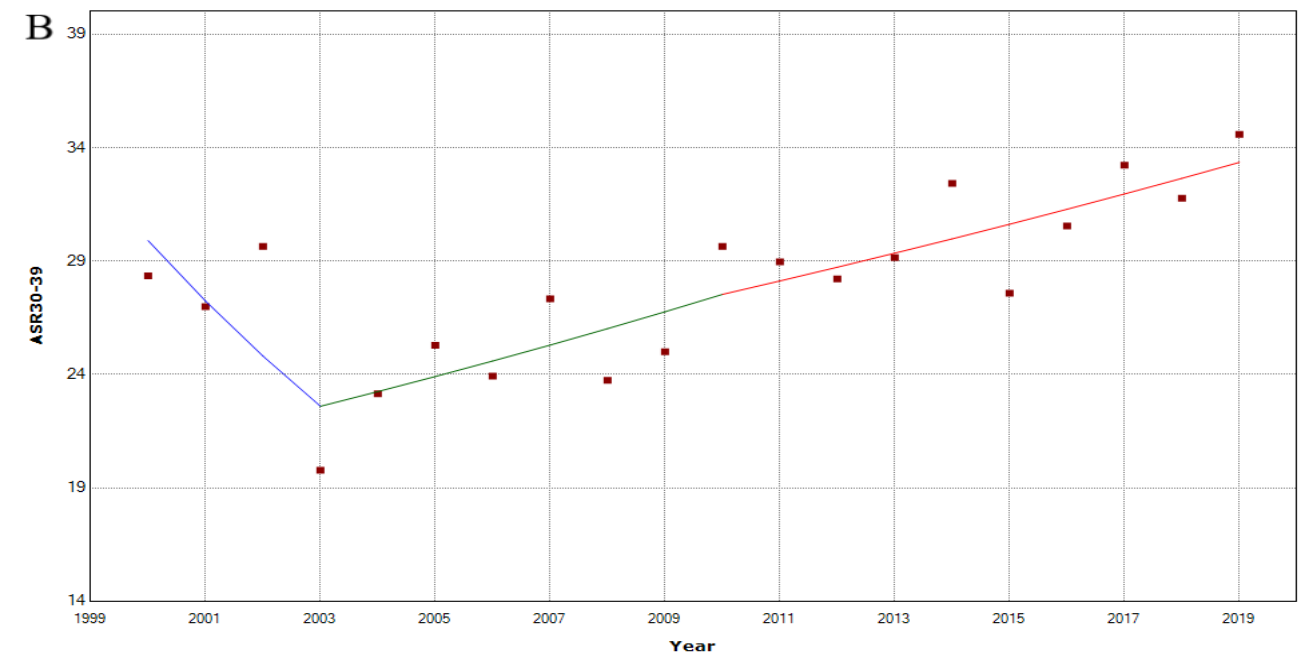

- Observed $-2000-2003 \mathrm{APC}=-8.91^{\circ}-2003-2010 \mathrm{APC}=2.86 \quad$ 2010-2019 APC $=2.15^{*}$

- Indicates that the Annual Percent Change (APC) is significantly different from zero at the alpha $=0.05$ level.
Final Selected Model: 1 Joinpoint.

All: 1 Joinpoint

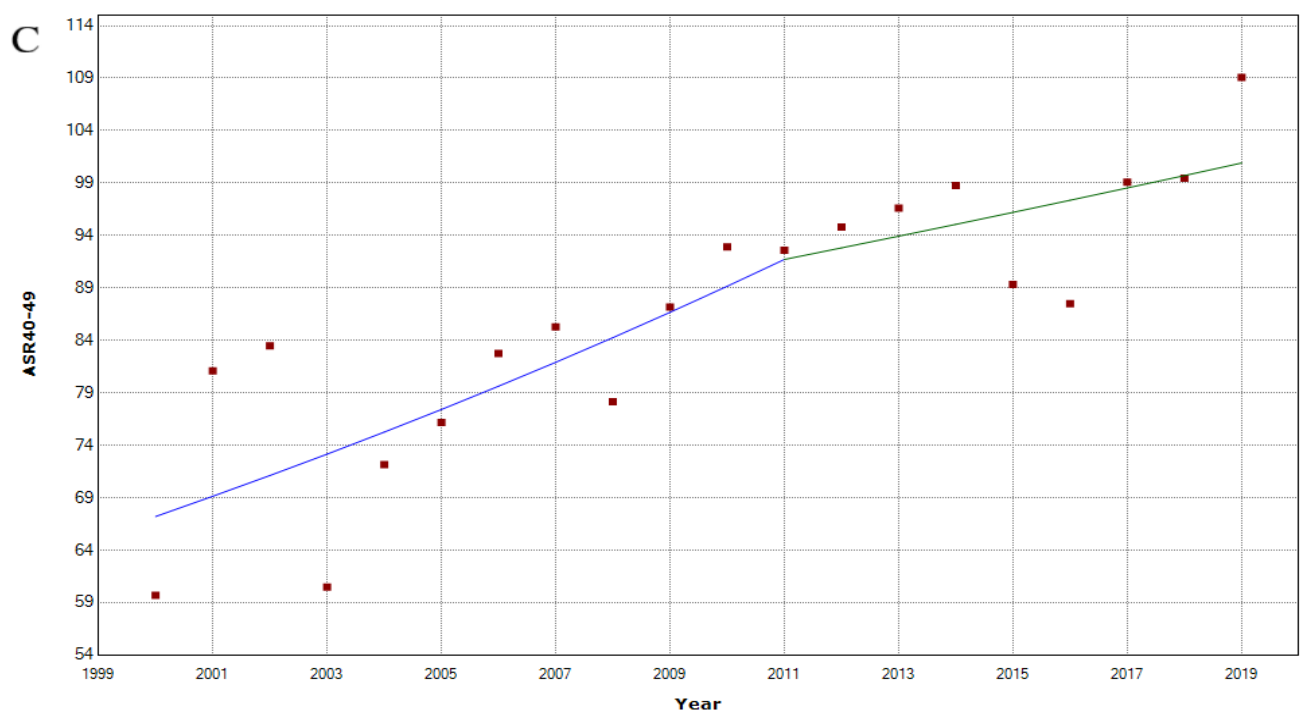

- Observed - 2000.000-2011.000 APC $=2.866^{*} \quad$ — 2011.000-2019.000 APC $=1.201$

- Indicates that the Annual Percent Change (APC) is significantly different from zero at the alpha $=0.05$ level.
Final Selected Model: 0 Joinpoints.

3892 Asian Pacific Journal of Cancer Prevention, Vol 22 
All: 2 Joinpoints

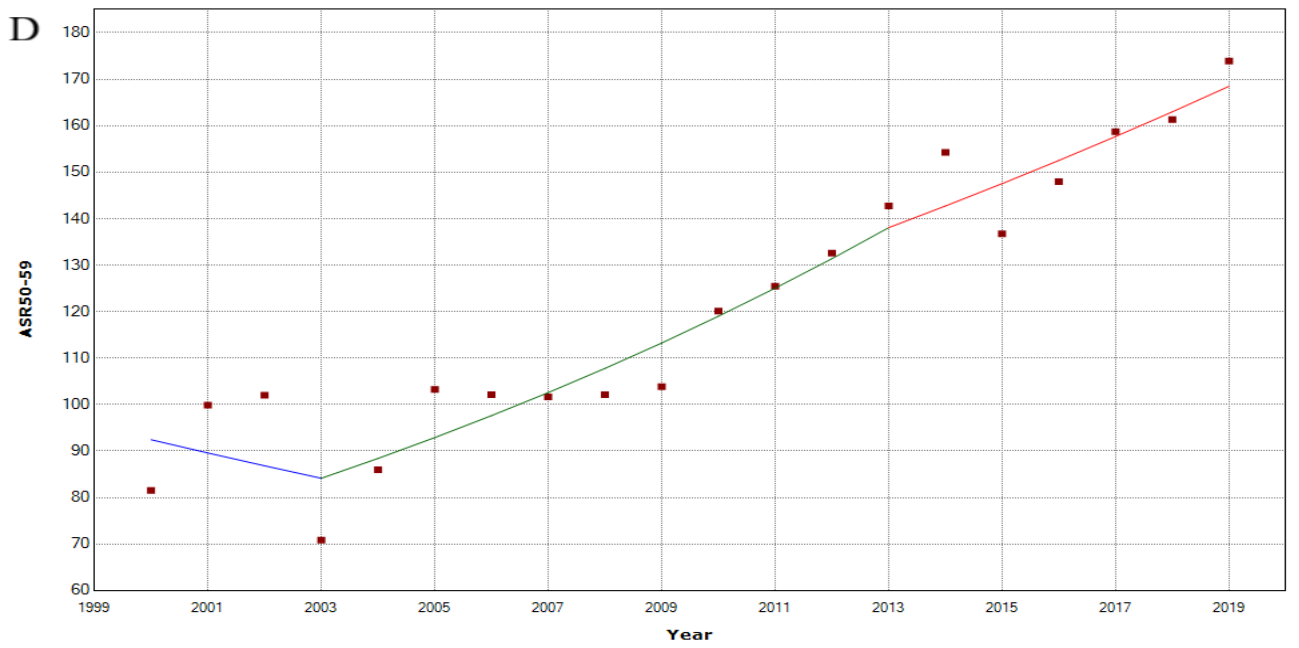

- Observed - 2000.000-2003.000 APC $=-3.089 \quad$-2003.000-2013.000 APC $=5.077^{\circ} \quad$ - 2013.000-2019.000 APC $=3.376^{\circ}$ - Indicates that the Annual Percent Change (APC) is significantly different from zero at the alpha $=0.05$ level
Final Selected Model: 0 Joinpoints.

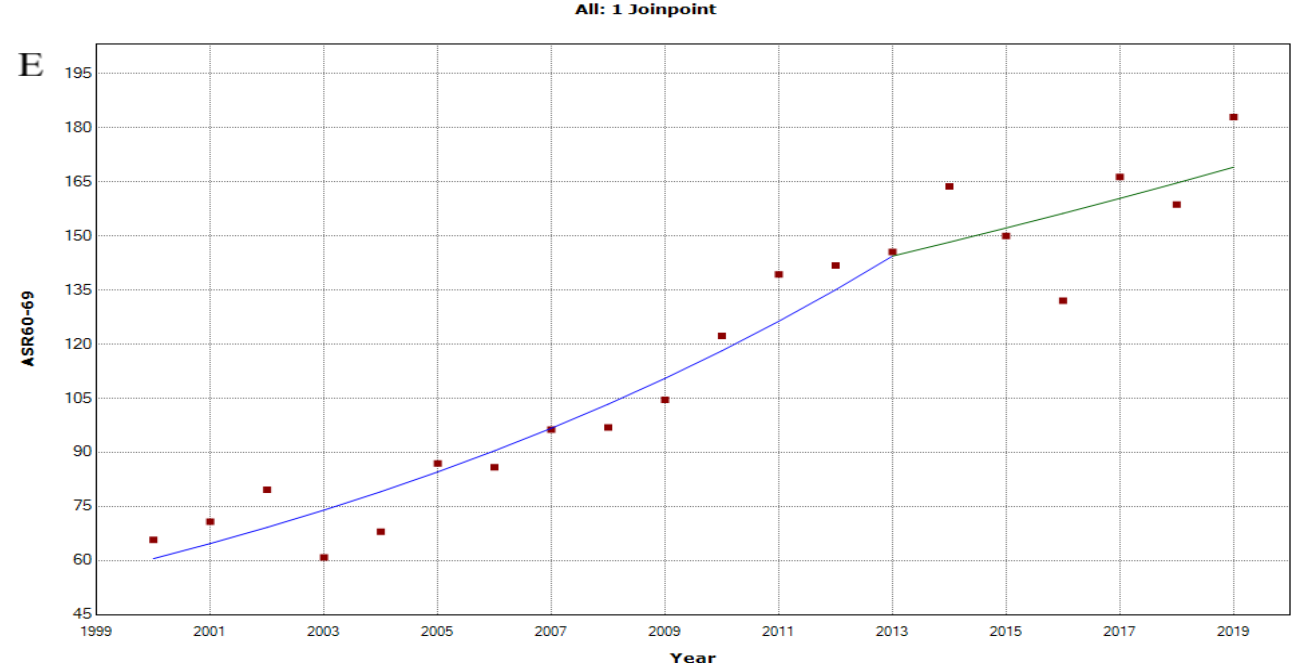

\begin{tabular}{|ll}
\hline- Observed $-2000.000-2013.000 \mathrm{APC}$ & $=6.913^{\circ}-2013.000-2019.000 \mathrm{APC}=2.660$ \\
\hline
\end{tabular}

- Indicates that the Annual Percent Change (APC) is significantly different from zero at the alpha $=0.05$ leve
Final Selected Model: 0 Joinpoints.

All: 1 Joinpoint

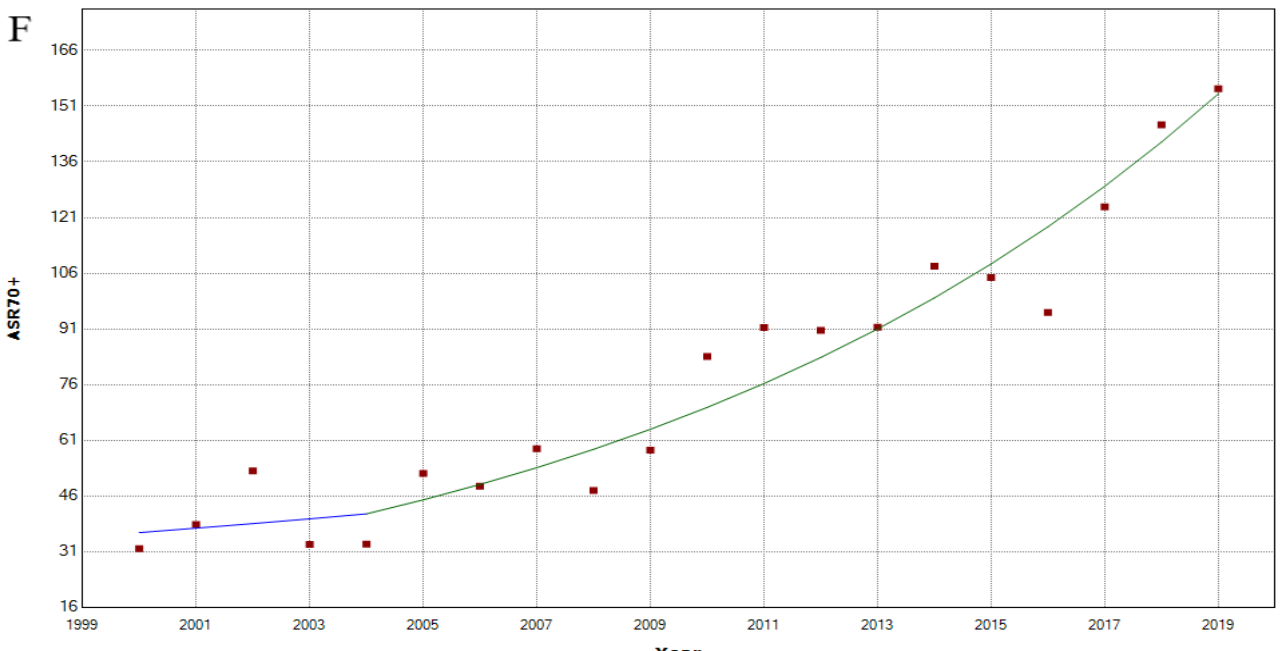

- Observed - 2000.000-2004.000 APC $=3.323 \quad$ - 2004.000-2019.000 APC $=9.210^{\circ}$

-Indicates that the Annual Percent Change (APC) is significantly different from zero at the alpha $=0.05$ level.
Einal Selected Model: 0 J loinpoint

Figure 2. Joinpoint regression analysis of age-specific incidence rates $(1 / 100,000)$ of breast cancer for Iraqi women, 2000-2019; age group (20-29), A; age group (30-39), B; age group (40-49), C; age group (50-59), D; age group (60$69)$, E; age group (70+), F, 


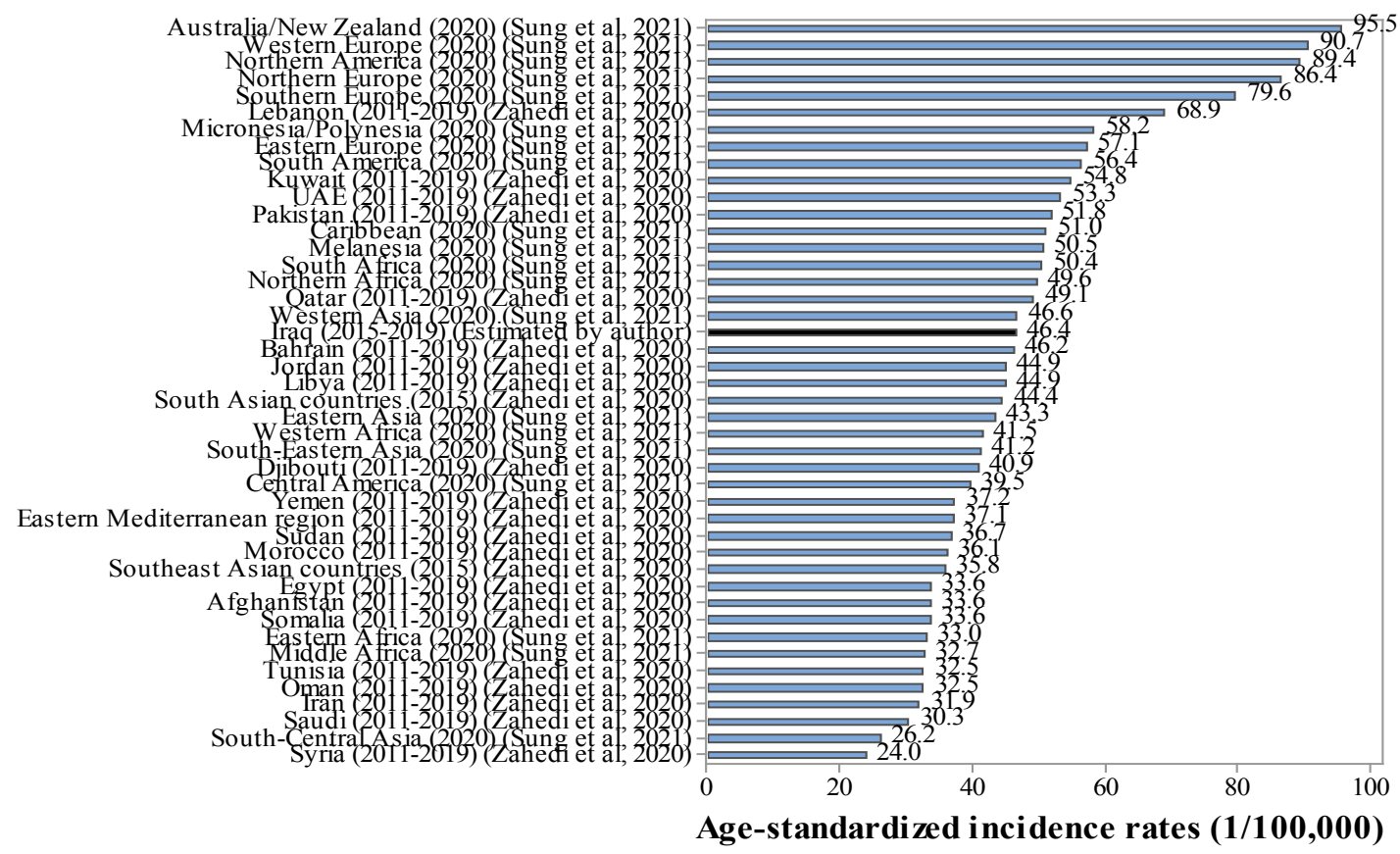

Figure 3. Age-Standardized Incidence Rates $(1 / 100,000)$ of Breast Cancer for Iraqi Women Compared to Other Countries.

to $-1.025 \%$ ), no joinpoint detected. In the age group 20-29 years, APC increased statistically not significant over the period 2000-2019 with AAPC $+0.986 \%(95 \%$ CI -5.859,+8.329). Two joinpoints were identified, 2003 and 2006; 2000-2003 (APC - $21.869 \%$; $95 \%$ CI -37.083 , $-2.976 \%$ ); $2003-2006$ (APC $+20.575 \% ; 95 \%$ CI -21.810 , $+85.938 \%) ; 2006-2019(\mathrm{APC}+2.852 \% ; 95 \% \mathrm{CI}+0.543$, $+5.213 \%)$. In the age group 30-39 years, AAPC not significantly increased from 2000 to 2019 with APC $+0.576 \%$ (95\% CI $-1.214 ;+2.399 \%)$, with two joinpoints were observed, 2003 and 2010; 2000-2003 (APC -8.911 $\%$; $95 \%$ CI $-16.852,-0.213 \%) ; 2003-2010$ (APC +2.862 $\% ; 95 \%$ CI $-0.262,+6.083 \%$ ); 2010-2019 (APC +2.153 $\% ; 95 \%$ CI $+0.466,+3.869 \%$ ). In the age group $40-49$ years, AAPC increased statistically significant from 2000 to 2019 with $\mathrm{APC}+2.162 \%(95 \% \mathrm{CI}+0.529 ;+3.822 \%)$. One joinpoint was detected in 2011; 2000-2011 (APC $+2.866 \%$; $95 \% \mathrm{CI}+0.870,+4.902 \%$ ), 2011-2019 (APC $+1.201 \%$; $95 \%$ CI $-1.958,+4.463 \%)$. The incidence significantly increased in the age group $50-59$ years during

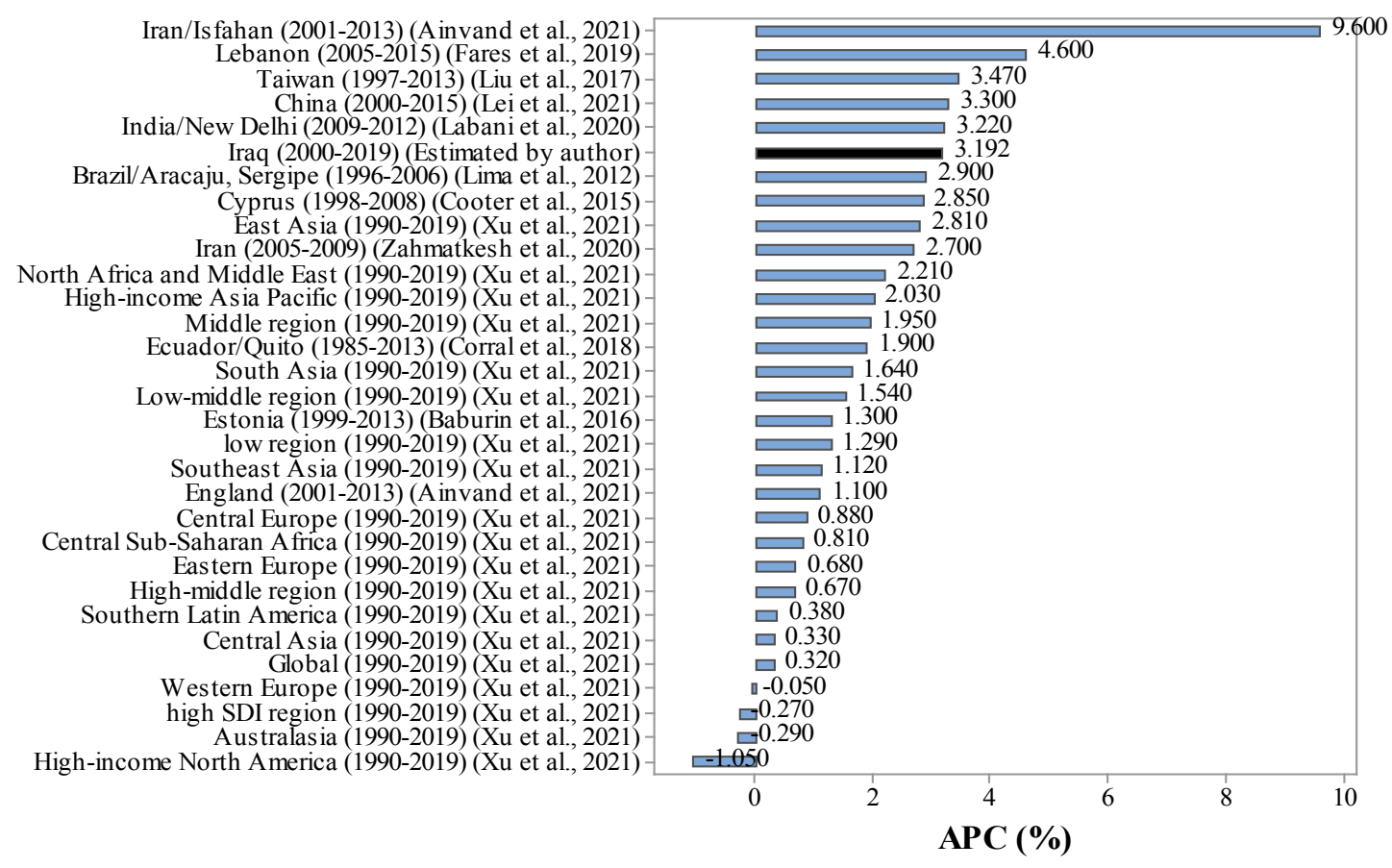

Figure 4. Annual Percent change (APC) of breast cancer for Iraqi women compared to other countries. 
2000-2019 with an AAPC of $+3.210 \%(95 \% \mathrm{CI}+1.408$, $+5.043 \%$ ). Two joinpoints were found, 2003 and 2013 (2000-2003: APC $-3.089 \%, 95 \%$ CI $-11.581,+6.218 \%$; 2003-2013: APC $+5.077 \%, 95 \% \mathrm{CI}+3.332,+6.851 \%$, and 2013-2019: APC $+3.376 \%, 95 \%$ CI $+0.220,+6.631 \%$, receptively). Statistically significant changes were found for the age group 60-69 years (AAPC, $+5.551 ; 95 \% \mathrm{CI}$, +3.586 to +7.555$)$. One joinpoint was found in 2013 ; 2000-2013 ( $\mathrm{APC}+6.913 \% ; 95 \% \mathrm{CI}+5.147,+8.710 \%$ ), 2013-2019 (APC $+2.660 \%$; 95\% CI $-2.711,+8.328 \%$ ). The highest incidence changes were found in the age group of 70+ over the period 2000-2019 with an AAPC of $+7.943 \%(95 \% \mathrm{CI}+4.589,+11.404 \%)$. One joinpoint was found in 2004; 2000-2004 (APC +3.323\%; 95\% CI $-10.691,+19.537 \%), 2004-2019$ (APC $+9.210 \%$; 95\% CI $+7.103,+11.358 \%$ ).

\section{Discussion}

This study examines how the incidence of breast cancer has changed over time (20 years) in Iraq. According to this study, the ASIR reached 52.70/100,000 in 2019, which is more than double the rate estimated in 2000 (24.364/100,000). There was a significantly increasing trend for breast cancer incidence over the whole studied period. The trend changes in the annual incidence between 2000 and 2019 raised by $116.301 \%$, and the changes in the period incidence between 2000-04 and 2015-19 raised by $76.509 \%$.

Breast cancer incidence rates are higher in most developed countries, due to its association with numerous risk factors (Menvielle et al., 2011) and the availability of regular screening strategies (Esserman et al., 2009). The highest rates $(>90 / 100,000)$ were found in Australia/ New Zealand $(95.5 / 100,000)$ and Western Europe $(90.7 / 100,000)$, while the lowest rates $(<30)$ were reported in South-Central Asia $(26.2 / 100,000)$ and Syria (24.0/100,000) (Figure 3). In Eastern Mediterranean region countries over the period 2011-2019, the highest rates were reported in Lebanon $(68.9 / 100,000)$, while the lowest rates were reported in Syria $(24.0 / 100,000)$. Iraq has a moderate rate of breast cancer incidence, similar to Western Asia, Qatar, Bahrain, and Jordan (Figure 3).

The wide geographical variation in the prevalence of risk factors (such as age at menarche, age at menopause, use of menopausal hormone therapy, age at first birth, Lifetime duration of breastfeeding, low parity, family history, alcohol consumption, smoking, obesity, or physical inactivity), prevention, diagnostic evaluation, and treatment, as well as disparities in data quality throughout the world, may partially explain the observed substantial between country and region differences in incidence from breast cancer (Shin et al., 2010; Leong et al., 2010; Barnard et al., 2015; Torre et al., 2017; Sung et al., 2021; Lima et al., 2021).

Since 1990, the global incidence of breast cancer has increased by $1.44 \%$ each year. Since 1990 , the incidence of breast cancer has increased considerably in all age categories, ranging from $1.28 \%$ per year (50-69) to $1.55 \% \%$ year (under 50 ). All regions have shown statistically significant increases in incidence. North Africa and the Middle East saw the highest per-year rise in overall incidence. Only North America has shown a reduction in the overall incidence (Lima et al., 2021).

Many studies in Iraq reported that the peak incidence of breast cancer occurs at older ages (Alwan et al., 2019; Majid and Hassan, 2017). According to this study, females over the age of 70 years in Iraq were at high risk of developing breast cancer, with an AAPC of $+7.943 \%$ from 2000 to 2019, while the APC from 2004 to 2019 was $+9.210 \%$. These effects are likely due to lifestyle, including increasing levels of overweight, dietary changes, physical inactivity (Lafta et al., 2013; Karim et al., 2015; Alwan et al., 2019; Shwana et al., 2020). In Iraq, most of the patients with breast cancer are diagnosed at a late stage (Alwan et al., 2019; Mutar et al., 2019). As a result, breast cancer public awareness campaigns should be promoted to avoid patient delays in presentation and to support the national breast cancer early detection program.

The observed breakpoint in 2003 linked, in part, to the 2003 Gulf war and extensive looting that began in the days following the US forces' invasion of Iraq, which resulted in the suspension of work in government offices as well as the destruction of official documents (Al-Hashimi and Wang, 2014). Over the study period, we determined a significant increasing trend for breast cancer incidence rates in Iraq, which higher in Middle-Eastern countries, and among several countries in the world (Figure 4).

However, it is beyond the scope of this article to investigate why there are increasing or decreasing trends in breast cancer incidence in Iraq. The advances in diagnosis and improved case reporting in recent years, availability of screening strategies in all Iraqi governorates' main hospitals, in addition to women's lifestyle changes, such as for overweight and obesity, physical inactivity, family history, delayed first birth, and not breastfeeding, may have led to an increase in the risk of breast cancer in Iraq.

In summary, our findings revealed an increase in the incidence rates of breast cancer among Iraqi women from 2000 to 2019. Further research is needed to explore risk factors including levels of overweight, dietary changes, physical inactivity, obesity, smoking, high marriage age, and low birth rates to prevent and control breast cancer policies.

\section{Author Contribution Statement}

None.

\section{Acknowledgments}

I would like to thank the Iraqi Cancer Board-Ministry of Health employees for their efforts in giving data access. I am grateful to the University of Mosul for supporting our work.

\section{Approval}

This paper was not carried out as part of a student project or thesis.

\section{Availability of data}

The paper was conducted based on the annual book Asian Pacific Journal of Cancer Prevention, Vol $22 \mathbf{3 8 9 5}$ 
series published by the Iraqi cancer registry/Ministry of Health. Iraqi Cancer Board each year published detailed statistical data on cancer in Iraq. Annual estimates of the population, by 5-year age groups and by gender obtained from the United Nations, Population Division, Department of Economic and Social Affairs.

\section{Conflict of interest}

I declare that I have no conflicts of interest to disclose regarding this manuscript.

\section{References}

Ainvand MH, Shakibaei N, Ravankhah Z, Yadegarfar Gl (2021). Breast cancer incidence trends in Isfahan province compared with those in England over the period 2001-2013. Int J Prev Med, 12, 12-54.

Al-Alwan NA, Mualla FH, (2014). Promoting clinical breast examination as a screening tool for breast cancer in Iraq. Iraqi Nat J Nurs Special, 27.

Al-Alwan NA, Tawfeeq FN, Mallah NA (2019). Demographic and clinical profiles of female patients diagnosed with breast cancer in Iraq. J Contemp Med Sci, 5, 14-9.

Al-Hashimi MM, Wang XJ (2014). Breast cancer in Iraq, incidence trends from 2000-2009. Asian Pac J Cancer Prev, 15, 281-6.

Baburin A, Aareleid T, Rahu M, Reedik L, Innos K (2016). Recent changes in breast cancer incidence and mortality in Estonia: transition to the west. Acta Oncol, 55, 728-33.

Barnard ME, Boeke CE, Tamimi RM (2015) Established breast cancer risk factors and risk of intrinsic tumor subtypes. Biochim Biophys Acta Rev Cancer, 1856, 73-85.

Cooter M, Soliman AS, Pavlou P, et al (2015). Incidence and time trends of cancer in Cyprus over 11 years (1998-2008), Tumori, 101, 8-15.

Corral Cordero F, Cueva Ayala P, Yépez Maldonado J, Tarupi Montenegro W (2018). Trends in cancer incidence and mortality over three decades in Quito-Ecuador, Colomb. Med, 49, 35-41.

Danaei G, Vander Hoorn S, Lopez AD, et al (2005). Comparative Risk Assessment collaborating group (Cancers. Causes of cancer in the world: comparative risk assessment of nine behavioural and environmental risk factors. Lancet, 366, 1784-93.

Esserman L, Shieh Y, Thompson I, (2009) Rethinking screening for breast cancer and prostate cancer. JAMA, 302, 1685-92.

Fares MY, Salhab HA, Khachfe HH, Khachfe HM (2019). Breast cancer epidemiology among Lebanese women: an 11-year analysis. Medicina, 55, 463.

Iraqi Cancer Board (2019). Results of the Iraqi Cancer Registry 2019. Baghdad, Iraqi Cancer Registry Center, Ministry of Health.

Karim SA, Ghalib HH, Mohammed SA, Fattah FH (2015). The incidence, age at diagnosis of breast cancer in the Iraqi Kurdish population and comparison to some other countries of Middle-East and West. Int J Surg, 13, 71-5.

Labani S, Asthana S, Srivastava A, Vohra P, Bhatia D (2020). Incidence and trends of breast and cervical cancers: A Joinpoint regression analysis. Indian J Med Paediatr Oncol, 41, 654-62.

Lafta RK, Isa SA, Saeed EQ (2013). Risk factors of breast cancer among women (A Sample from Baghdad, Iraqi J Med Sci, 26.

Lei S, Zheng R, Zhang S, et al (2021). Breast cancer incidence and mortality in women in China: temporal trends and projections to 2030. Cancer Biol Med, 18, 900-9.

Leong SP, Shen ZZ, Liu TJ, et al (2010). Is breast cancer the same disease in Asian and Western countries?. World J Surg, 34, 2308-24.

Lima CA, Rangel MR, Macedo-Lima M, da Silva AM (2012). Time trends in breast cancer incidence and mortality in a mid-sized northeastern Brazilian city. BMC Public Health, 12, 1-7.

Lima SM, Kehm RD, Terry MB (2021). Global breast cancer incidence and mortality trends by region, age-groups, and fertility patterns. E Clin Med, 38, 100985.

Liu FC, Lin HT, Kuo CF, et al (2017). Epidemiology and survival outcome of breast cancer in a nationwide study. Oncotarget, 8, 16939-50.

Majid RA, Hassan HA, Muhealdeen DN, Mohammed HA, Hughson MD (2017). Breast cancer in Iraq is associated with a unimodally distributed predominance of luminal type B over luminal type A surrogates from young to old age. $B M C$ Womens Health, 17, 1-8.

Menvielle G, Kunst AE, Van Gils CH, et al (2011). The contribution of risk factors to the higher incidence of invasive and in situ breast cancers in women with higher levels of education in the European prospective investigation into cancer and nutrition. Am J Epidemiol, 173, 26-37.

Mutar MT, Goyani MS, Had AM, Mahmood AS (2019). Pattern of presentation of patients with breast cancer in Iraq in 2018: A cross-sectional study. J Glob Oncol, 5, 1-6.

Shin HR, Joubert C, Boniol M, et al (2010). Recent trends and patterns in breast cancer incidence among Eastern and Southeastern Asian women. Cancer Causes Control, 21, 1777-85.

shwana Shwana JJ, Gad ZM, Abd Elhafeez SS, et al (2020). An epidemiological study of female breast cancer in Sulaymaniyah City, Iraqi Kurdistan. Tabari Biomed Stu Res $J, 2020$.

Sung H, Ferlay J, Siegel RL, et al (2021). Global cancer statistics 2020: GLOBOCAN estimates of incidence and mortality worldwide for 36 cancers in 185 countries. CA Cancer J Clin, 71, 209-49.

Torre LA, Islami F, Siegel RL, Ward EM, Jemal A (2017). Global cancer in women: burden and trends. Cancer Epidemiol Biomark Prev, 26, 444-57.

Xu S, Liu Y, Zhang T, et al (2021). The global, regional, and national burden and trends of breast cancer from 1990 to 2019: Results From the Global Burden of Disease Study 2019. Front Oncol, 11, 1789.

Zahedi R, Vardanjani HM, Baneshi MR, et al (2020). Incidence trend of breast cancer in women of eastern Mediterranean region countries from 1998 to 2019: A systematic review and meta-analysis. BMC Womens Health, 20, 1-10.

Zahmatkesh B, Keramat A, Alavi N, et al (2016). Breast cancer trend in Iran from 2000 to 2009 and prediction till 2020 using a trend analysis method. Asian Pac J Cancer Prev, 17, 1493-8.

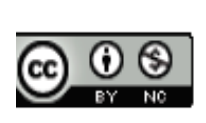

This work is licensed under a Creative Commons AttributionNon Commercial 4.0 International License. 